\title{
Use of GC and PDSC methods to characterize human milk fat substitutes obtained from lard and milk thistle oil mixtures
}

\author{
Joanna Bryś $^{1}$ - Inês Filipa Vaz Flores ${ }^{2} \cdot$ Agata Górska $^{1} \cdot$ Magdalena Wirkowska-Wojdyła $^{1}$. \\ Ewa Ostrowska-Ligęza ${ }^{1} \cdot$ Andrzej Brys $\hat{s}^{3}$
}

Received: 1 December 2016/ Accepted: 9 May 2017/Published online: 26 May 2017

(c) The Author(s) 2017. This article is an open access publication

\begin{abstract}
The aim of this study was the analytical evaluations of human milk fat substitutes (HMFS) synthesized via enzymatic interesterification of lard and milk thistle oil mixtures by a immobilized commercial sn-1,3-specific lipase, using calorimetric and chromatographic methods. The mixtures of lard and milk thistle oil at mass ratio 6:4 and 8:2 were interesterified for 2,4 and $6 \mathrm{~h}$ at the temperature of $60{ }^{\circ} \mathrm{C}$. The determination of fatty acid composition was carried out by gas chromatographic analysis of fatty acid methyl esters. The positional distribution of fatty acids in the sn-2 and sn-1,3 positions of triacylglycerols was based on the ability of the pancreatic lipase to selectively hydrolyze ester bonds in the sn-1,3 positions. Pressure differential scanning calorimetry (PDSC) method was used for the determination of the oxidative stability of HMFS. The oxidative induction time was obtained from the PDSC curves. Due to enzymatic interesterification of mixtures of lard and milk thistle oil, new HMFS that have a similar regiospecific structure of triacylglycerols to human milk fat can be produced. The induction time obtained
\end{abstract}

The results of this research were presented at the 11th Seminar to the memory of prof. Stanislaw Bretsznajder.

Joanna Bryś

joanna_brys@sggw.pl

1 Faculty of Food Sciences, Warsaw University of Life Sciences (WULS - SGGW), Nowoursynowska st. 159c, 02-787 Warsaw, Poland

2 Faculty of Biotechnology, Catholic University of Portugal, Dr. António Bernardino de Almeida st., 4200-072 Porto, Portugal

3 Faculty of Production Engineering, Warsaw University of Life Sciences (WULS - SGGW), Nowoursynowska st. 164, 02-787 Warsaw, Poland from PDSC measurements can be used as a parameter for the assessment of the resistance of tested fats to their thermal-oxidative decomposition.

Keywords Lard · Milk thistle oil - PDSC · Interesterification - Oxidative stability · Human milk fat substitutes

\section{Abbreviations}

L Lard

MTO Milk thistle oil

LM6:4_2 Mixture of L and MTO in proportions 6:4, interesterified for $2 \mathrm{~h}$ at $60^{\circ} \mathrm{C}$

LM8:2_2 Mixture of L and MTO in proportions 8:2, interesterified for $2 \mathrm{~h}$ at $60^{\circ} \mathrm{C}$

LM6:4_4 Mixture of L and MTO in proportions 6:4, interesterified for $4 \mathrm{~h}$ at $60{ }^{\circ} \mathrm{C}$

LM8:2_4 Mixture of L and MTO in proportions 8:2, interesterified for $4 \mathrm{~h}$ at $60^{\circ} \mathrm{C}$

LM6:4_6 Mixture of L and MTO in proportions 6:4, interesterified for $6 \mathrm{~h}$ at $60^{\circ} \mathrm{C}$

LM6:4_6 Mixture of L and MTO in proportions 8:2, interesterified for $6 \mathrm{~h}$ at $60{ }^{\circ} \mathrm{C}$

HMF Human milk fat

\section{Introduction}

There is the commonly accepted opinion that breastfeeding is recognized to enable the best development and growth of newborns; however, infant formulas became an alternative among mothers who cannot breastfeed their baby. The development of infant milk fat similar to human milk fat (HMF) catches the attention and became a challenge for food industry [1]. Modified lipids resembling the 
triacylglycerols (TAG) of human milk can be produced by chemical or enzymatic modification of TAG, but in the past few years enzymatic modification of TAG has prevailed. According to previous reports [1-3], enzymatic acidolysis and interesterification using sn-1,3-specific lipases are strategies that have received considerable attention in the literature. In contrast to chemical methods, enzymatic synthesis as a tool for fat modification has many advantages. Catalyzed with lipases, interesterification reactions can be directed in controlled ways, and specific or totally new products can be produced [4]. Microbial lipases have a great potential for commercial application due to their stability, selectivity and broad substrate specificity. Lipasecatalyzed reactions are carried out under milder conditions and with a higher selectivity than chemically catalyzed reactions. The use of 1,3-selective lipases allows to maintain the fatty acids in the sn-2 position of the acylglycerols. This is nutritionally desirable and not possible to attain by chemical catalysis $[3,5]$.

Breast milk is considered the main source of nutrients and energy for many infants in their early life because of nutritional, immunological and food safety points of view. Lipids in human milk provide a major source of energy and essential structural components for the cell membranes of the newborn [2, 6]. Human milk consists of $3-5 \%$ of lipids and approximately $99 \%$ of them are TAG. Palmitic acid (16:0), oleic acid (18:1) and linoleic acid $(18: 2 n-6)$ are the three major fatty acids of TAG, which constitutes 25,30 and $15 \%$ of total fatty acids, respectively [7]. HMF similarly to other milk fats contains high content of the saturated palmitic acid, but its unique feature is also a high content of the polyunsaturated fatty acids (PUFA) [8, 9]. The polyunsaturated fatty acid components of human milk are complex, including both the C18 precursors, linoleic acid (18:2 n-6) and $\alpha$-linolenic acid (18:3n-3), and the bioactive very long-chain polyunsaturated fatty acids (LCPUFA) of both the $n-6$ and the $n-3$ families [10]. The major LCPUFA in HMF are eicosapentaenoic acid (C20:5n-3, EPA), docosahexaenoic acid (C22:6n-3, DHA) and arachidonic acid (C20:4n-6, ARA). ARA and DHA are membrane fatty acids required for growth and development of the central nervous system of infants. Recent studies also show the importance of ARA and DHA for bone mineralization and bone mass, probably an effect caused by the influence of the fatty acids on biosynthesis of prostaglandins. Therefore, infant formula should also contain these LCPUFA in appropriate amounts [11]. The structure of human milk TAG is also unique, as $60-70 \%$ of palmitic acid is located at the sn-2 position and the sn- 1 and sn- 3 positions are taken by 18:0, 18:1 and 18:2 fatty acids [12]. This intramolecular structure is an advantageous aspect for the efficiency of calcium absorption [7].
Therefore, HMF is the component that supplies not only the highest fraction of the infant's required dietary energy [13]. The specific fatty acid compositions and structure of HMF provide also an efficient fat absorption by infants. The main goals of production of the HMFS were to obtain fatty acid composition and positional acyl distribution as close as possible to those found in HMF [1].

In order to produce HMFS that mimic HMF, several studies have been carried out using immobilized lipases as catalysts. In the majority of these studies, HMFS resembling HMF were obtained by enzymatic interesterification or acidolysis reactions of tripalmitin or lard with free fatty acids from different sources. Lard has been recommended as a potential alternative because it is the only fat resembling HMF in terms of fatty acid composition [14]. Compared with HMF, lard has a similar palmitic and oleic acids content but is characterized by lower content of essential fatty acids. Vegetable oils like milk thistle oil are a source of PUFA. A combination of tripalmitin or lard with vegetable oils has been chosen to obtain a product possessing similar fatty acids composition and their distribution in TAG to HMF $[2,5,15]$.

As Nielsen et al. [12] supposed, for new HMFS products which are in development, it is necessary to optimize the nutritional and physical properties as well as ensure that they possess acceptable oxidation stability. Lipids are one of the mostly chemically unstable food components, and when lipid oxidation occurs, it gives rise to the formation of lipid hydroperoxides. Free fatty acids (FFA) and formation of lipid hydroperoxides increase the formation of secondary volatile products of oxidation that influence unpleasant odor. In order to maintain a healthy product, it is important that lipids are oxidatively stable [12]. Differential scanning calorimetry (DSC) is one of the most popular methods for the appraisal of the oxidative stability of oils and fats [16]. It is based on measuring of the induction times of oils oxidation. The peroxide value (PV) is widely used for measuring primary products of oxidation.

The aim of this study was the analytical evaluations of HMFS synthesized via enzymatic interesterification of lard and milk thistle oil mixtures by a immobilized commercial sn-1,3-specific lipase, using calorimetric and chromatographic methods.

\section{Materials and methods}

\section{Chemicals and materials}

All the solvents and reagents were purchased from Avantor Performance Materials Poland S.A. (Gliwice, Poland) except for porcine pancreatic lipase (Type II) which was 
supplied together with the standard compounds by SigmaAldrich (Saint Louis, MO, USA). Immobilized Lipozyme RM IM, used in this investigation as a catalyst of interesterification, was also procured from Sigma-Aldrich. Lipozyme RM IM is a food-grade granulated silica preparation of a microbial 1,3-specific lipase from Rhizomucor miehei (activity $150 \mathrm{IUN} \mathrm{g}^{-1}$ ). The silica gel TLC plate was purchased from Merck (Darmstadt, Germany). All the solvents and reagents used in analyses were of chromatographic or analytical grade. Lard and milk thistle oil were provided by commercial company. The mixtures of L and MTO in mass proportions $6: 4$ or $8: 2$ were used in this investigation.

\section{Enzymatic interesterification}

Flasks containing of the initial mixtures were prepared and positioned in a thermostated mineral-oil bath shaker. After thermal equilibration of sample at $60{ }^{\circ} \mathrm{C}$, the enzymatic catalyst $(8 \% \mathrm{w} / \mathrm{w})$ was added. The interesterifications were performed with continuous shaking and stopped by filtering off the biocatalyst after a predetermined time (2, 4 or $6 \mathrm{~h})$.

\section{Acid values and free fatty acids content}

Acid value (AV) was determined by titration of fat samples with $0,1 \mathrm{M}$ ethanolic potassium hydroxide solution. Fatty acid concentration was calculated based on acid values and the value of molar mass of oleic acid. Acids values were determined according to ISO method 660:2000 [17].

\section{Peroxide value}

The peroxide value (PV) of mixture was determined by iodometric technique with visual endpoint detection in accordance with Standards ISO 3960:2007 [18].

\section{DSC measurements}

A differential scanning calorimeter (DSC Q20 TA) coupled with a high-pressure cell (PDSC-pressure differential scanning calorimetry) was used. The instrument was calibrated using high-purity indium as a standard. Fat samples of 3-4 mg were weighted into an aluminum open pan and placed in the sample chamber under oxygen atmosphere with an initial pressure of $1400 \mathrm{kPa}$ and with the $100 \mathrm{~mL} \mathrm{m^{-1 }}$ oxygen flow rate. The isothermal temperature for each sample was $120^{\circ} \mathrm{C}$. Obtained diagrams were analyzed using TA Universal Analysis 2000 software. For each sample, the output was automatically recalculated and presented as amount of energy per one gram. The maximum PDSC oxidation time (induction time) was determined based on the maximum rate of oxidation (maximum rate of heat flow).

\section{Fatty acid composition / GC measurements}

The determination of fatty acid composition was carried out by gas chromatographic (GC) analysis of fatty acid methyl esters. Methyl esters of fatty acids were prepared through transesterification with sodium methoxide according to ISO 5509:2001. An YL6100 GC chromatograph equipped with a flame ionization detector and BPX-70 capillary column of $0.20 \mathrm{~mm}$ i.d. $\times 60 \mathrm{~m}$ length and $0.25 \mu \mathrm{m}$ film thickness was used. The oven temperature was programmed as follows: $60{ }^{\circ} \mathrm{C}$ for $5 \mathrm{~min}$ and then it was increased by $10^{\circ} \mathrm{C} \min ^{-1}$ to $180{ }^{\circ} \mathrm{C}$; from 180 to $230{ }^{\circ} \mathrm{C}$, it was increased by $3{ }^{\circ} \mathrm{C} \min ^{-1}$ and then kept at $230{ }^{\circ} \mathrm{C}$ for $15 \mathrm{~min}$. The temperature of the injector was $225^{\circ} \mathrm{C}$, with a split ratio of $1: 100$, and the detector temperature was $250{ }^{\circ} \mathrm{C}$. Nitrogen flowing with the rate of $1 \mathrm{~mL} \mathrm{~min}^{-1}$ was used as the carrier gas. The results were expressed as relative percentages of each fatty acid (\% of the fatty acids peaks area was calculated). Fatty acids were identified by comparing the relative retention times of FAME peaks with FAME chemical standard.

\section{Positional distribution of fatty acids in the sn-2 and sn-1,3 positions of TAG}

The positional distribution of fatty acids in the sn- 2 and sn1,3 positions of TAG is based on the ability of the pancreatic lipase to selectively hydrolyze ester bonds in the sn1,3 positions. Briefly, $20 \mathrm{mg}$ of purified pancreatic lipase (porcine pancreatic lipase, crude type II), $1 \mathrm{~mL}$ of Tris buffer ( $\mathrm{pH} 8.0), 0.25 \mathrm{~mL}$ of bile salts $(0.05 \%)$ and $0.1 \mathrm{~mL}$ of calcium chloride $(2.2 \%)$ were added to $50 \mathrm{~mL}$ centrifuge tubes and vortexed with $0.1 \mathrm{~g}$ of fat sample. The mixture was incubated at $40{ }^{\circ} \mathrm{C}$ in a water bath for $5 \mathrm{~min}$ and then $1 \mathrm{~mL}$ of $6 \mathrm{~mol} \mathrm{~L}^{-1} \mathrm{HCl}$ and $1 \mathrm{~mL}$ of diethyl ether were added and the mixture was centrifuged. Diethyl ether layer was collected to test tubes and evaporated under nitrogen gas to obtain $200 \mathrm{uL}$ volume. A $200 \mathrm{uL}$ aliquot was loaded onto a silica gel TLC plate with fluorescent indicator $254 \mathrm{~nm}$ and developed with hexane/diethyl ether/acetic acid (50:50:1, v:v:v). The 2-monoacylglycerol (2-MAG) band was visualized under UV light. The 2-MAG band was scraped off into a screw-capped test tube, extracted twice with $1 \mathrm{~mL}$ of diethyl ether and centrifuged. The ether layer was collected and entirely evaporated under nitrogen, and then the sample was dissolved in n-hexane and methylated as described above. 


\section{Statistical analysis}

Relative standard deviation was calculated, where appropriate, for all data collected using Microsoft Excel 2012 software. One-way analysis of variance ANOVA was performed using the Statgraphics Plus, version 5.1. Differences were considered to be significant at a p-value of 0.05 , according to Tukey's multiple range test.

\section{Results and Discussion}

\section{Fatty acids composition and their distribution in TAG in studied fats}

HMF contains a similar amount of monounsaturated fatty acids (MUFA) and saturated fatty acids (SFA), 39.1 and $40.6 \%$ respectively, and about $19.5 \%$ of PUFA [19]. Lard, which was the subject of research, is a rich source of SFA, among palmitic acid $(23.0 \%)$ and stearic acid (14.0\%). It also contains oleic acid (46.1\%) belonging to MUFA and linoleic acid (9.7\%) belonging to PUFA in significant amounts. These 4 major fatty acids match approximately $92.8 \%$ of the total fatty acids. The fatty acid compositions of lard are in agreement with results published by LopezLopez [19]. Comparing to HMF, lard is characterized by a similar SFA content (about 39.5\%), but higher MUFA and less PUFA content, 49.7 and $10.7 \%$, respectively (Fig. 1). The fatty acids composition of milk thistle oil was different compared to lard. Linoleic acid in milk thistle oil accounts for $52.0 \%$ of the total fatty acids, while oleic acids content reached $26.6 \%$. The other fatty acid was palmitic acid which constitutes only $7.6 \%$ of the total fatty acids. Analyzing the fatty acids profile of the milk thistle oil, PUFA constitute about $55.1 \%$ of the total fatty acids, which is a higher amount compared to HMF that contains only $19.5 \%$ of PUFA. On the other hand, the SFA and MUFA content were lower compared to HMF, 17.1 and $27.8 \%$ respectively.

In order to enrich TAG derived from L in PUFA, the enzymatic interesterification was applied. As a source of PUFA, milk thistle oil was used. As a result of interesterification, the essential fatty acids from milk thistle oil were incorporated into TAG structures of lard. The interesterified fats contained from 19.5 to $31.3 \%$ of PUFA (Table 1; Fig. 1). Mixtures after interesterification showed from 17.9 to $29.0 \%$ of linoleic acid belonging to omega- 6 essential fatty acid, while the original lard only $9.7 \%$ of this acid was observed. TAG of interesterified fats contained also from 0.5 to $0.9 \%$ of $\alpha$-linolenic acid belonging to omega-3 essential fatty acid. The enzymatic interesterification of lard and thistle oil blends were performed at various proportions and times to obtain a fatty acid profile matching that of HMF. The SFA content in the blends after interesterification ranged from 26.9 to $36.6 \%$, the MUFA from 39.9 to $50.7 \%$ and PUFA from 19.4 to $28.9 \%$. In general, percentage of SFA in all mixtures after interesterification was lower in comparison with that of HMF (40.6\%). The MUFA contents in the blends of lard and oil mixed in proportions 6:4 after interesterification were about 39\%, and these values were similar to those of to HMF (39.1\%). In the interesterified blend of lard and oil mixed in proportions $8: 2$, similar amount of PUFA $(19.5-22.3 \%)$ was detected compared to the reference HMF (19.5\%). The blends of lard and oil mixed in proportions 8:2 after 2 and $6 \mathrm{~h}$ of reaction were characterized by higher amount of MUFA, about $50.7 \%$, compared to the reference HMF $(39.1 \%)$. In the blends of lard and vegetable oil mixed in proportions 6:4, the PUFA content was higher than in the mixture 8:2 after interesterification. The results obtained in this study suggest that in blends after interesterification no significant differences in the fatty acid profile were
Fig. 1 Content of fatty acids (SFA saturated fatty acids, MUFA monounsaturated fatty acids, PUFA polyunsaturated fatty acids) for mixtures after interesterification and for human milk fat. *Human milk fat. Values are derived from Lopez-Lopez et al. [19]

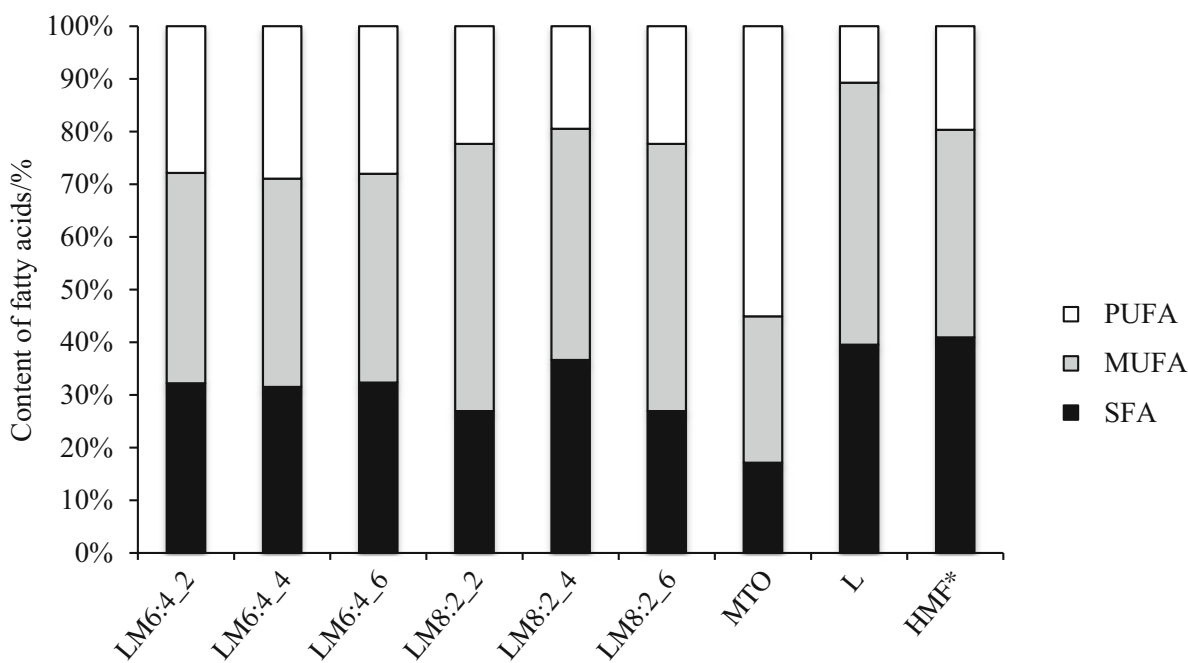


Table 1 Fatty acids composition (\%) of mixtures after interesterification

\begin{tabular}{|c|c|c|c|c|c|c|}
\hline Fatty acid & LM6:4_2 & LM6:4_4 & LM6:4_6 & LM8:2_2 & LM8:2_4 & LM8:2_6 \\
\hline C10:0 & 0.0 & 0.0 & 0.0 & 0.1 & 0.1 & 0.1 \\
\hline C12:0 & 0.0 & 0.0 & 0.1 & 0.1 & 0.1 & 0.1 \\
\hline C14:0 & 0.8 & 0.8 & 0.8 & 1.2 & 1.1 & 1.2 \\
\hline C16:0 & 17.5 & 17.1 & 17.7 & 23.6 & 21.0 & 23.6 \\
\hline $\mathrm{C} 16: 1 \mathrm{c}$ & 1.3 & 1.3 & 1.3 & 2.0 & 1.8 & 2.0 \\
\hline C17:0 & 0.2 & 0.2 & 0.2 & 0.3 & 0.3 & 0.3 \\
\hline $\mathrm{C} 17: 1 \mathrm{c}$ & 0.2 & 0.2 & 0.2 & 0.2 & 0.2 & 0.2 \\
\hline C18:0 & 11.5 & 11.2 & 11.3 & 0.0 & 13.1 & 0.0 \\
\hline $\mathrm{C} 18: \ln 9 \mathrm{c}$ & 37.4 & 37.0 & 37.1 & 47.3 & 40.8 & 47.3 \\
\hline $\mathrm{C} 18 \mathrm{~s} 3 \mathrm{n} 3 \mathrm{c}$ & 25.9 & 26.9 & 26.2 & 20.7 & 17.9 & 20.7 \\
\hline C20:0 & 0.6 & 0.7 & 0.6 & 0.9 & 0.8 & 0.9 \\
\hline $\mathrm{C} 20: 1 \mathrm{c}$ & 1.9 & 1.8 & 1.8 & 1.2 & 1.1 & 1.2 \\
\hline $\mathrm{C} 21: 0$ & 1.1 & 1.1 & 1.0 & 1.3 & 1.1 & 1.3 \\
\hline $\mathrm{C} 20: 1 \mathrm{c}$ & 0.4 & 0.3 & 0.3 & 0.5 & 0.0 & 0.5 \\
\hline $\mathrm{C} 20: 3 \mathrm{n} 3 \mathrm{c}$ & 1.3 & 1.3 & 1.2 & 0.8 & 0.7 & 0.8 \\
\hline
\end{tabular}

observed independently of the processes conditions used (time of interesterification). HMF contains also long-chain polyunsaturated fatty acids (LCPUFA) 20:4n-6 and 22:6n3 . Nevertheless, these fatty acids were not found in blends after interesterification. However, these mixtures contain other essential fatty acids like linoleic acid and $\alpha$-linoleic acid, which can be converted in our organism to LCPUFA.

The type of fatty acid and its stereospecific position in TAG determine both, the physical behavior of dietary fats and their fate during digestion and absorption [20] Considering the results obtained, it can be concluded that interesterification affects the distribution of fatty acids in TAG in both, internal and external positions. HMFS are characterized by a similar fatty acid composition in sn-2 position of TAG, regardless of time of process. Palmitic acid was the most abundant fatty acid located in sn-2 position of interesterified mixtures. The reaction time influenced to a small extent to reduce the palmitic acid content and increase in the stearic and oleic acid content in sn-2 position of TAG. Due to the positional sn-1,3 specifity of the lipase, the interesterification catalyzed by Lipozyme RM IM occurred mainly in external positions of TAG. As the enzyme operated on the external positions, the percentages of given fatty acids in sn-2 positions of interesterified TAG in comparison with their counterparts for initial blends remain nearly unchanged [21]. The changes in sn-2 percentages can be caused by possible acyl migrations in TAG molecules during prolonged time of interesterification, as it was proved by $\mathrm{Xu}$ et al. [22]. Increasing the amount of oil in the sample from 20 to $40 \%$ caused the reduction in the palmitic acid content in sn-2 position of TAG from $44.8-50.9$ to $34.7-36.4 \%$.
However, in order to obtain the confirmation of fatty acid distribution between regiospecific TAG positions, it was essential to recalculate the fatty acid composition of sn-2 position into relative fatty acid in sn-2 position [19]. As reported by Lopez-Lopez et al. [19], fatty acid composition of sn-1,3 and sn-2 in TAG positions is not the most suitable way of presenting data of sn-2 fatty acid composition without taking into account the total percentage of fatty acids in whole TAG molecules. Hence, Fig. 2 presents the percentage of most abundant fatty acids in sn-2 position of TAG, expressed as relative fatty acid in analyzed fats. Human milk contains approximately $70 \%$ of palmitic acid in the sn-2 position, whereas most animal and vegetable fats contain this fatty acid primarily in the sn-1 and sn-3 positions [12]. Lard, like HMF, contains approximately $85.7 \%$ of palmitic acid in the sn-2 position of TAG, whereas percentage of this fatty acid in internal position of TAG in oil reached only $14.6 \%$. Analyzing the results regarding the percentage of the fatty acids esterified at sn- 2 position of TAG in HMFS, it can be concluded that the structure of TAG is very similar to the HMF. Percentage of the palmitic acids at sn-2 of TAG in all HMFS exceeded $33 \%$, which means that it is located mainly in the internal position of TAG. This location increases the efficiency of calcium absorption by infants. Taking into account the percentage of unsaturated fatty acids in the sn-2 position of TAG in HMFS, it can be stated that they are located mostly in external positions of TAG. This is mainly oleic acid. Percentage of oleic acid at the sn- 2 of TAG in HMFS ranged from 14.1 to $22.8 \%$, which confirms that it is located mainly in sn-1,3 positions of TAG. 


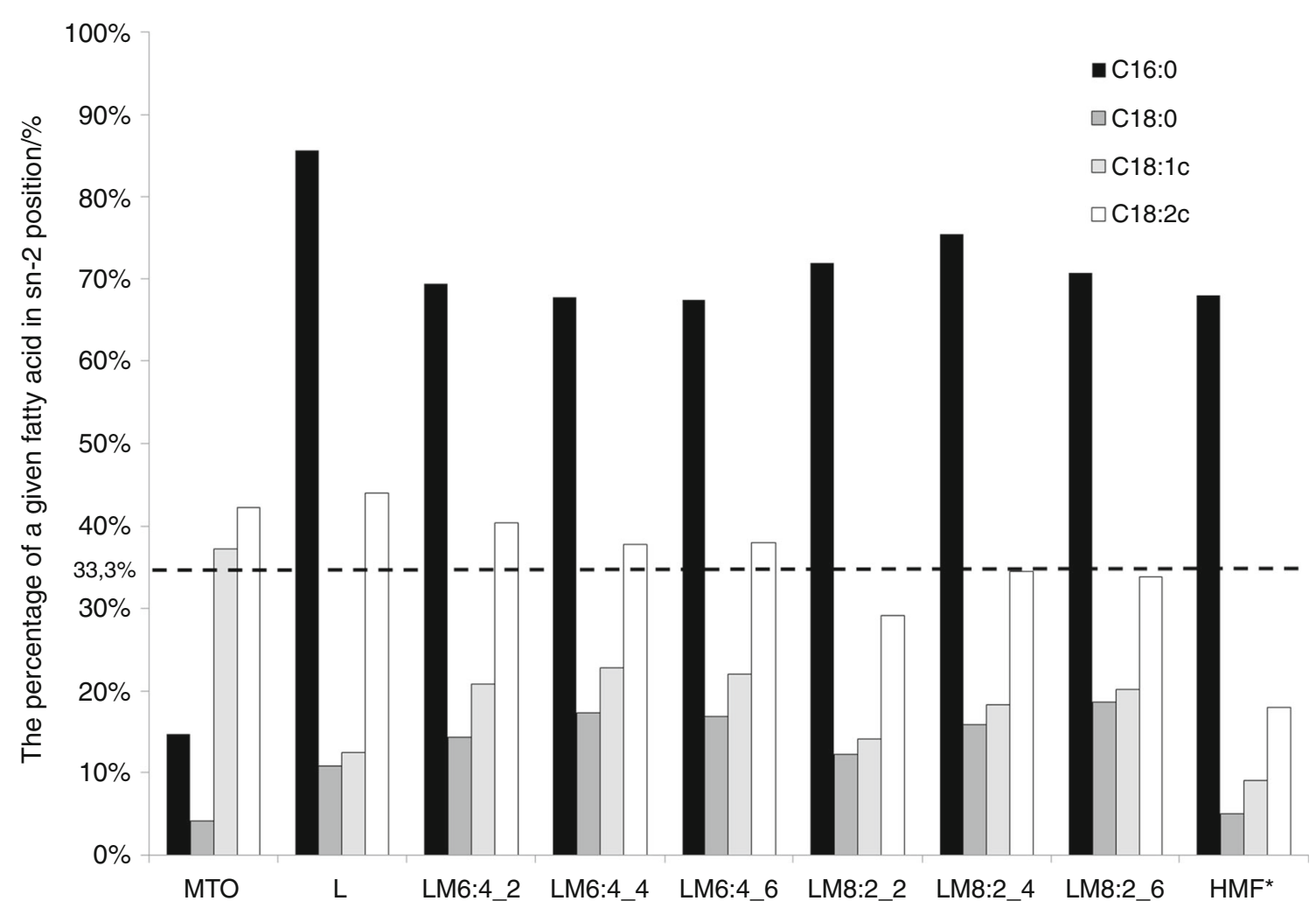

Fig. 2 Percentage of a given fatty acid in sn-2 position of TAG of interesterified fats. *Human milk fat. Values are derived from Lien et al. [10]

Table 2 Acid value, peroxide value and oxidation induction time of raw materials and mixtures after interesterification

\begin{tabular}{llll}
\hline $\begin{array}{l}\text { Type of } \\
\text { sample }\end{array}$ & $\begin{array}{l}\text { PV/ } \\
\mathrm{mmol} \mathrm{O}_{2} \mathrm{~kg}^{-1}\end{array}$ & \multicolumn{1}{l}{$\begin{array}{l}\mathrm{AV} / \\
\mathrm{mg} \mathrm{KOH} \mathrm{g}\end{array}$} & \multicolumn{1}{l}{ FFA/\% } \\
\hline MTO & $5.84 \pm 0.41^{\mathrm{c}}$ & $2.44 \pm 0.10^{\mathrm{b}}$ & $1.23 \pm 0.05^{\mathrm{b}}$ \\
L & $0.72 \pm 0.13^{\mathrm{a}}$ & $1.13 \pm 0.18^{\mathrm{a}}$ & $0.57 \pm 0.09^{\mathrm{a}}$ \\
LM6:4_2 & $1.44 \pm 0.31^{\mathrm{b}}$ & $12.40 \pm 0.01^{\mathrm{d}}$ & $6.23 \pm 0.01^{\mathrm{d}}$ \\
LM6:4_4 & $0.72 \pm 0.05^{\mathrm{a}}$ & $12.38 \pm 0.88^{\mathrm{d}}$ & $6.22 \pm 0.44^{\mathrm{d}}$ \\
LM6:4_6 & $0.84 \pm 0.10^{\mathrm{a}}$ & $16.22 \pm 0.73^{\mathrm{e}}$ & $8.15 \pm 0.37^{\mathrm{e}}$ \\
LM8:2_2 & $0.84 \pm 0.32^{\mathrm{a}}$ & $10.20 \pm 0.16^{\mathrm{c}}$ & $5.13 \pm 0.08^{\mathrm{c}}$ \\
LM8:2_4 & $0.41 \pm 0.13^{\mathrm{a}}$ & $13.19 \pm 0.78^{\mathrm{d}}$ & $6.63 \pm 0.39^{\mathrm{d}}$ \\
LM8:2_6 & $0.60 \pm 0.08^{\mathrm{a}}$ & $15.44 \pm 0.11^{\mathrm{e}}$ & $7.76 \pm 0.06^{\mathrm{e}}$
\end{tabular}

Data expressed as mean \pm standard deviation

The different lowercase letters (a-e) in the same column indicate significantly different values $(p<0.05)$

\section{The quality assessment of studied fats}

All fats and oils are susceptible to oxidation processes, giving rise to free radicals, hydroperoxides and polymers [23]. The peroxide value is a method used for determining the amount of oxygen chemically bound to an oil or fat as peroxides, particularly hydroperoxides which are primary oxidation products. The PV of the raw materials and HMFS are listed in Table 2. The blends after interesterification were characterized by lower PV, in contrary to milk thistle oil, for which the parameter reached the values greater than 5.8 meq $\mathrm{O}_{2} \mathrm{~kg}^{-1}$ fat. High PV means a high production of hydroperoxides, which can alter or even lose the nutritional quality of fats. The products obtained by enzymatic reactions which were carried out for $2 \mathrm{~h}$ were characterized by the higher PV which means a faster progression of oxidation. Comparing the different proportions of substrates but maintaining the same time, HMFS obtained from interesterified mixture of lard and oil in ratio of $8: 2$ showed lower PV than the samples with a ratio of $6: 4$. This is the consequence of the fact that the samples with ratio of $6: 4$ contained bigger amount of oil which oxidizes quickly.

Therefore, all blends after interesterification showed a PV quite similar and lower than 2 meq $\mathrm{O}_{2} \mathrm{~kg}^{-1}$ fat. The HMFS were characterized by lower PV than oil and that made them more stable to oxidation. However, many studies have reported a decrease in oxidative stability of HMFS compared to initial fats $[1,23]$. The evidence about oxidative stability of HMFS is diverse; the main factors that determine the oxidative stability of HMFS are the different analytical methods of purification and production, the different nature of oil and lard sources and the presence of antioxidants during the production [23].

The main components of fats are TAG. Fats also contain certain quantities of monoacylglycerols, diacylglycerols 
and fatty acids contents. The determinant of free fatty acids contents in fat is acid value (AV). The AV and free fatty acids content (FFA) of lard, milk thistle oil and blends after interesterification are shown in Table 2. Analyzing the results, it can be observed that $\mathrm{AV}$ of mixtures after interesterification was higher compared to lard and oil, which indicate a bigger presence of FFA. Lard was characterized by the lowest FFA content $(<1 \%)$, similarly to studied vegetable oil with an amount of FFA at the level of $<2 \%$. Analyzing and comparing the results, the FFA content in mixtures increased after enzymatic interesterification reaction. These results are in agreement with Brys et al. [14], who indicated that enzymatic interesterification process caused the increase in FFA amount. The $\mathrm{AV}$ and FFA contents depend significantly on the time of the reaction. In the case of interesterified mixtures, the AV was lower when reaction time was shorter $(\sim 2 \mathrm{~h})$. General blends of lard and milk thistle oil after interesterification at the presence of Lipozyme RM IM contained $5.1-8.2 \%$ of FFA. Therefore, it was demonstrated that increase in AV is related to increase in FFA content in the sample. Hamam and Shahidi [24] suggested that the presence of FFA in the reaction mixture may induce oxidation due to a catalytic effect of the carboxylic groups of the fatty acids contents on the formation of free radicals. In general, the higher the level of fatty acids contents, monoacylglycerols and diacylglycerols in the obtained product after interesterification with respect to the level of TAG is, the higher is the reduced oxidative stability [23]. It should be concluded that before using HMFS for potential applications in functional foods, the FFA should be removed.
Numerous methods have been developed for monitoring fat and oil autoxidation [25-28]. DSC is a very popular method for the assessment of the oxidative stability of oils and fats. The DSC experiments are performed with a linear increase in temperature (dynamic conditions) or at constant temperature (isothermal conditions). The oxidation medium (oxygen or air) can be maintained at normal (atmospheric) pressure or at increased pressure (PDSC) [16]. The results of PDSC measurements expressed as the oxidation induction times are shown in Fig. 3. The induction times obtained for analyzed fats can be used as primary parameters for the assessment of the resistance of tested fats to their oxidative decomposition. Mostly, samples with longer induction time are more stable than those for which the induction time obtained is shorter [21]. The PDSC tests for HMFS performed at isothermal temperature of $120{ }^{\circ} \mathrm{C}$ showed that their induction times (28.9-34.8 $\mathrm{min}$ ) were reduced compared with the lard (46.8 min). Comparing raw materials, milk thistle oil was characterized by lower induction time $(32.7 \mathrm{~min})$ than lard. In general, oils contain a high concentration of unsaturated fatty acids and they have worse oxidative stability, consequently a short induction time. Comparing the proportions between mixtures of lard and milk thistle oil, the induction time should be lower in samples with ratios of 6:4. This is a result of higher concentration of oil unsaturated fatty acids. Most studies have reported a decrease in oxidative stability of interesterified fats, especially fats rich in unsaturated fatty acids, compared to the raw materials $[1,23,29]$. Same studies have suggested also the loss of antioxidants during the production of
Fig. 3 Oxidation induction time of interesterified fats and raw materials. Different letters indicate that the samples are significantly different at $p<0.05$

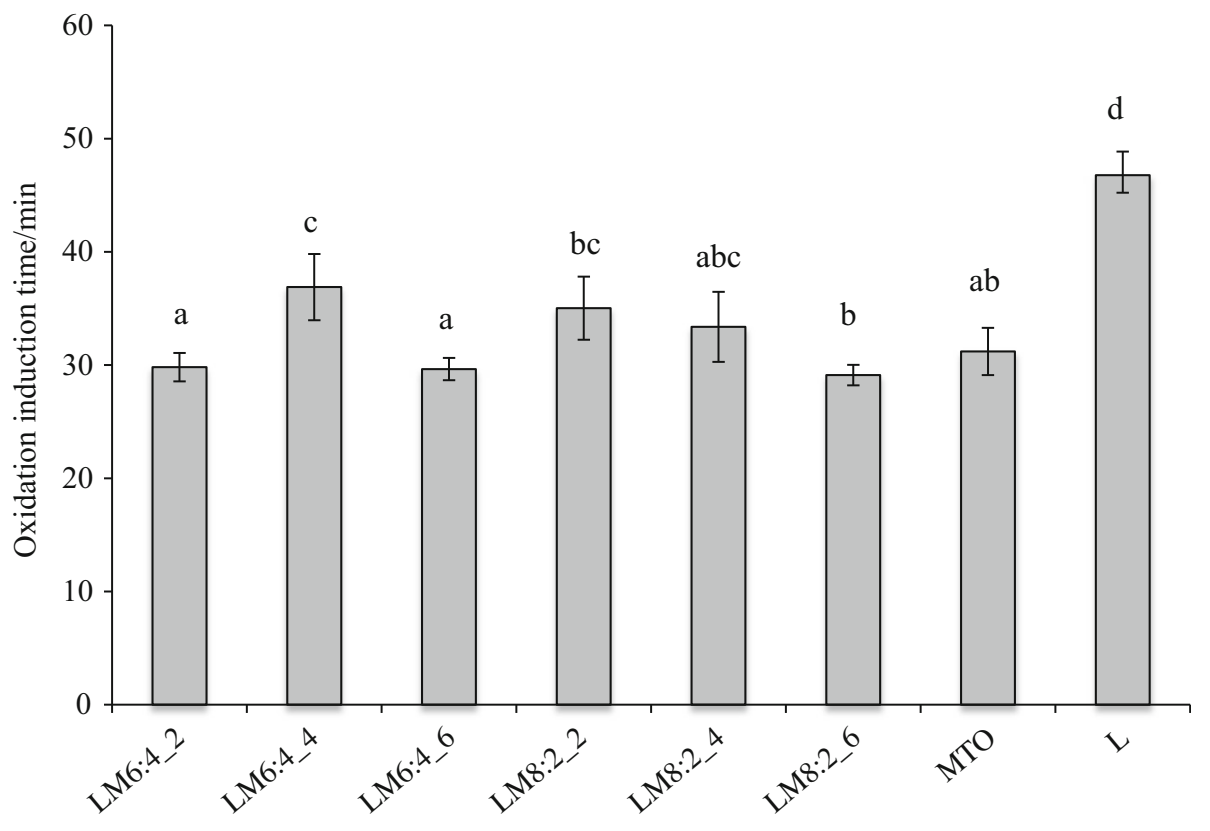


interesterified fats as the main reason for explaining a worse oxidative stability found for fats after modification even regardless of the production method used or of the polyunsaturated nature of fatty acids. This loss of endogenous antioxidants is especially important when vegetable oils are used as a source of fatty acids for the production of interesterified fats, due to their natural content in antioxidants, such as tocols, both tocopherols and tocotrienols, phytosterols, or phenolic compounds [23].

\section{Conclusions}

The interesterified mixtures of L and MTO were satisfactory to produce HMFS rich in PUFA. The essential fatty acids from MTO were incorporated into TAG structures of lard after modification. Major fatty acids determined in the studied fats after interesterification were palmitic, oleic and linoleic acids, similarly to HMF. The interesterified blends were characterized also by a similar ratio of SFA, MUFA and PUFA like HMF, especially blend of lard and oil mixed in proportions $8: 2$ after $4 \mathrm{~h}$ of reaction.

The oxidative stability of the HMFS showed that products based on blends of lard and milk thistle oil after interesterification were more stable to oxidation than oil. The interesterified fats showed a short induction time compared to lard, which means their worse oxidative stability. PDSC is a fast and reliable method that can be used to assess the oxidation parameters of interesterified mixtures of lard and milk thistle oil. The induction times obtained for analyzed fats can be used as primary parameters for the assessment of the resistance of tested fats to their oxidative decomposition. The products of enzymatic reactions which were carried out for $2 \mathrm{~h}$ underwent faster progression of oxidation. This means a worse oxidative stability for blends which were carried out during short time. The mass ratio of substrates in blends also influenced the oxidative stability. In blends which contained a higher amount of vegetable oil, the progression of oxidation was faster.

In general, FFA content of mixture increased after interesterification reaction. The presence of free fatty acids after the reaction can decrease the oxidative stability of interesterified fats. It should be concluded that before using HMFS for potential applications in functional foods, the FFA should be removed.

Positional distribution of fatty acids in the TAG of interesterified blends was similar to that of HMF. The sn1,3 positions predominantly contain unsaturated FA, mostly oleic acid and sn-2 position contain mostly palmitic acid. This position of palmitic acid in TAG is important for proper absorption of fat and minerals by infants.
Open Access This article is distributed under the terms of the Creative Commons Attribution 4.0 International License (http://crea tivecommons.org/licenses/by/4.0/), which permits unrestricted use, distribution, and reproduction in any medium, provided you give appropriate credit to the original author(s) and the source, provide a link to the Creative Commons license, and indicate if changes were made.

\section{References}

1. Maduko C, Park Y, Akoh CC. Characterization and oxidative stability of structured lipids: infant milk fat analog. JAOCS. 2008;85:197-204.

2. Yang T, Xu X, He C, Li L. Lipase-catalyzed modification of lard to produce human milk fat substitutes. Food Chem. $2003 ; 80: 473-81$.

3. Wang YH, Qin XL, Zhu QS, Zhou R, Yang B, Li L. Lipasecatalyzed acidolysis of lard for the production of human milk fat substitute. Eur Food Res Technol. 2010;230:769-77.

4. Forssell P, Kervinen R, Lappi M, Linko P, Suoptti T, Poutanen K. Effect of enzymatic interesterification on the melting point of tallow-rapeseed oil (LEAR) mixture. JAOCS. 1992;69:126-9.

5. Tecelao C, Silva J, Dubreucq E, Ribeiro M, Ferreira-Dias S. Production of human milk fat substitutes enriched in omega-3 polyunsaturated fatty acids using immobilized commercial lipases and Candida parapsilosis lipase/acyltransferase. J Mol Cat B Enzym. 2010;65:122-7.

6. Jensen RG. The lipids in human milk. Prog Lipid Res. 1996;35:53-92.

7. $\mathrm{Mu} \mathrm{H}$. Production and nutritional aspects of human milk fat substitutes. Lipid Technol. 2010;22:126-9.

8. Sahin N, Akoh CC, Karaali A. Enzymatic production of human milk fat substitutes containing $\gamma$-linolenic acid: optimization of reactions by response surface methodology. JAOCS. 2005;82(8):549-57.

9. Xu X. Production of specific-structured triacylglycerols by lipasecatalyzed reactions: a review. Eur J Lip Sci Technol. 2000;102:287-303.

10. Lien EL. The role of fatty acid composition and positional distribution in fat absorption in infants. J Pediatr. 1994;125:562-8.

11. Huiling M. Production and nutritional aspects of human milk fat substitutes. Lipid Technol. 2010;22:126-9.

12. Nielsen NS, Yang $\mathrm{T}, \mathrm{Xu} \mathrm{X}$, Jacobsen $\mathrm{Ch}$. Production and oxidative stability of a human milk fat substitute produced from lard by enzyme technology in a pilot packed-bed reactor. Food Chem. 2006;94:53-60.

13. Sahin N, Akoh CC, Karaali A. Enzymatic production of human milk fat substitutes containing $\gamma$-linolenic acid: optimization of reactions by response surface methodology. JAOCS. 2005;82(8):549-57.

14. Bryś J, Wirkowska M, Górska A, Ostrowska-Ligęza E, Bryś A. Application of the calorimetric and spectroscopic methods in analytical evaluation of the human milk fat substitutes. J Therm Anal Calorim. 2014;118:841-8.

15. Ilyasoglu $\mathrm{H}$. Production of human fat milk analogue containing $\alpha$-linolenic acid by solvent-free enzymatic interesterification. LWT Food Sci Technol. 2013;54:179-85.

16. Ostrowska-Ligęza E, Bekas W, Kowalska D, Lobacz M, Wroniak M, Kowalski B. Kinetics of commercial olive oil oxidation: dynamic differential scanning calorimetry and Rancimat studies. Eur J Lipid Sci Technol. 2010;112:268-74.

17. ISO 660:2009. Animal and vegetable fats and oils-determination of acid value and acidity. International Organization for Standardization, Geneva, Switzerland. 
18. ISO 3960:2007. Animal and vegetable fats and oils-determination of peroxide value-iodometric (visual) endpoint determination. International Organization for Standardization, Geneva, Switzerland.

19. Lopez-Lopez A, Castellote-Bargalló AI, Campoy-Folgoso C, Rivero-Urgel M, Lopez-Sabater MC. Fatty acid and sn-2 fatty acid composition in human milk from Granada (Spain) and infant formulas. Eur J Clin Nutr. 2002;56:1242-54.

20. Small D. The effects of glyceride structure on fat adsorption and metabolism. Ann Rev Nutr. 1991;11:412-34.

21. Kowalski B, Tarnowska K, Gruczynska E, Bekas W. Chemical and enzymatic interesterification of beef tallow and rapeseed oil blend with low content of tallow. J Oleo Sci. 2004;53(10):479-88.

22. Xu X, Skands ARH, Adler-Nissen J, Høy CE. Production of specific structured lipids by enzymatic interesterification: optimization of the reaction by response surface design. Fett/Lipid. 1998;100(10):463-71.

23. Martin D, Reglero G, Senorans FJ. Oxidative stability of structured lipids. Eur Food Res Technol. 2010;231:635-53.
24. Hamam F, Shahidi F. Enzymatic acidolysis of an arachidonic acid single-cell oil with capric acid. JAOCS. 2004;81:887-92.

25. Gray JI. Measurement of lipid oxidation: a review. JAOCS. 1978;55:539-46.

26. Kowalska D, Gruczynska E, Kowalska M. The effect of enzymatic interesterification on the physico-chemical properties and thermo-oxidative stabilities of beef tallow stearin and rapeseed oil blends. J Therm Anal Calorim. 2015;120:507-17.

27. Ratusz K, Popis E, Ciemniewska-Żytkiewicz H, Wroniak M. Oxidative stability of camelina (Camelina sativa L.) oil using pressure differential scanning calorimetry and Rancimat method. J Therm Anal Calorim. 2016;126:343-51.

28. Souza AL, Martínez FP, Ferreira SB, Kaiser CR. A complete evaluation of thermal and oxidative stability of chia oil. J Therm Anal Calorim. 2017;. doi:10.1007/s10973-017-6106-x.

29. Bryś J, Wirkowska M, Górska A, Ostrowska-Ligęza E, Bryś A, Koczoń P. The use of DSC and FT-IR spectroscopy for evaluation of oxidative stability of interesterified fats. J Therm Anal Calorim. 2013;113:481-7. 